\title{
DENIS: A DEEP NEAR INFRARED SOUTHERN SKY SURVEY
}

\section{N. EPCHTEIN}

Observatoire de Paris, Meudon, France

\section{Aim of the Project and Achieved Performances}

The aim of DENIS is to map the southern sky in three near-infrared photometric bands $\left(\mathrm{I}, \mathrm{J}, \mathrm{K}_{s}\right)$ at the $1 \mathrm{mJy}$ sensitivity level $\left(14^{\text {th }}\right.$ magnitude in $\left.\mathrm{K}_{s}\right)$ and arcsecond resolution using the ESO 1 meter telescope at La Silla, Chile. The DENIS operations have started in December 1995 after a protosurvey period of one year mainly dedicated to technical tests and pilot surveys of selected regions of high astronomical interest. In September 1996, approximately 3000 square degrees of the sky have been covered, and the survey should be completed in 1999 .

The main specifications of the project have been described several times (e.g., Epchtein et al. 1994; Copet 1996; Epchtein 1997) and the achieved performances are summarized in Table 1 . The dedicated 3 channel camera and its data handling hardware and software have been designed, implemented and tested by a European consortium of laboratories coordinated by the Département de Recherche Spatiale of Paris Observatory at Meudon. Important parts of the data acquisition routines were also developed at the University of Innsbruck, Austria.

\section{Data Processing}

The DENIS data processing is performed routinely in 2 dedicated data analysis centers (DAC) located at Paris (PDAC) and Leiden (LDAC). The PDAC processes and archives the raw images and implements an extended source database. The LDAC tasks (Deul et al. 1995) consist in source extraction and photometric calibration, and implementation of a point source database. Cross-identifications with other catalogs will be performed in collaboration with the Centre de Données Stellaires in Strasbourg. Data will be released in stages to the astronomical community one year after being 
TABLE 1. Main specifications of the DENIS cameras

\begin{tabular}{lccc}
\hline Channel & $I$ & $J$ & $K_{s}$ \\
\hline Central wavelength $(\mu \mathrm{m})$ & 0.8 & 1.25 & 2.15 \\
Array manufacturer & Tektronix & Rockwell & Rockwell \\
Size (nb. of pixels) & $1024 \times 1024$ & $256 \times 256$ & $256 \times 256$ \\
Pixel size ( $\mu$ m, arcsec) & 24,1 & 40,3 & 40,3 \\
Array quantum efficiency (aver.) & 0.65 & 0.81 & 0.61 \\
Read-out noise ( $\left.e^{-}\right)$ & 6.7 & 38 & 39 \\
Read-out time $($ second) & 2.98 & 0.13 & 0.13 \\
Exposure time (second) & 9 & 10 & 10 \\
Achieved limiting magnitude & & & \\
(point source 3 $\sigma$ ) & 18.5 & 16.3 & 14 \\
Magnitude of saturation & 9.5 & 8.5 & 6.5 \\
\hline
\end{tabular}

archived in the DACs. A first set of data will be widely released in Spring 1997.

\section{Science with DENIS}

The primary aim of DENIS is to provide astronomers with reference documents (catalogs, atlases, databases) that will be fully available by the end of 2000. In the meantime, scientific programs will benefit of parts of this wealth of new data. Based on the pilot program and first months of observations, several investigations have been performed and have already produced significant scientific results. Some are presented at this Symposium and briefly outlined hereafter.

Multicolour surveys in a spectral domain never explored at this scale and at this level of sensitivity, provide an exceptional opportunity to probe the stellar content of the Galaxy. Lower interstellar extinction and high sensitivity to evolved stars are two major advantages of the near-infrared surveys with respect to optical Schmidt plates. Two main programs are underway in these area, one focuses on the exploration of the galactic disk, and more specifically of the anticenter regions, the other on the bulge. Ruphy et al. (1996) have shown that the DENIS data can be used to determine with a good accuracy the cutoff of the stellar distribution and the scale length (see her paper, this volume, p. 231). Another project, called ISOGAL and managed by A. Omont, combines ISO and DENIS images taken in the galactic bulge. The purpose of this investigation is to probe the bulge populations at $15 \mu \mathrm{m}$ with ISOCAM in selected areas within the longitude range $-45^{\circ}$ to $+45^{\circ}$ at low latitude and to carry out systematic cross-identifications 
of ISO and DENIS sources. Colour diagrams involving DENIS and ISO data are successfully used to select evolved giant populations and recently formed stars (Pérault et al. 1996)

Search for young stars in nearby molecular complexes is also a domain in which near-infrared surveys will provide worthwhile data. A study of the Orion $A$ region has been performed by Copet (1996) which led to the discovery of a large number of new candidates of $\mathrm{T}$ Tau stars. A program aimed at finding the infrared counterparts of CS cores is in progress (see his paper, this volume, p. 172). Other interesting regions such as the Chameleon cloud are also under investigations, also in connection with ISOCAM observations.

\section{Acknowledgements}

The instrument team involves scientists and engineers from Paris Observatory, Paris Institut d'Astrophysique, and Innsbruck University (B. de Batz, P. Fouqué, S. Kimeswenger, F. Lacombe, T. Le Bertre, S. Pau, D. Rouan, J.C. Renault, D. Tiphène). The data analysis centers are managed by G. Simon and J. Borsenberger in Paris and E. Deul in Leiden with the contribution of T. Forveille from Grenoble Observatory. All the contributors to DENIS are warmly thanked. The observations are collected at the European Southern Observatory, La Silla, Chile. The DENIS project is funded partly by the SCIENCE and the Human Capital and Mobility plans of the European Commision under grants CT920791 and CT940627, by the European Southern Observatory, and by various national sources of funding.

\section{References}

Copet E., 1996, PhD. dissertation, Paris 6 , Le relevé infrarouge DENIS: mise en oeuvre de l'instrument et étude de la région de formation d'étoiles d'Orion $\mathrm{A}$

Deul E.R., et al., 1995, Proc. Euroconference on "Near-Infrared Sky Surveys", San Miniato, Italy, P. Persi, W.B. Burton, N. Epchtein, A. Omont (eds.), reprinted from Mem. S. A. It. Vol. $66-3$, p. 549

Epchtein N., et al., 1994, Astrophys. Sp. Sc. 217, 3

Epchtein N., 1997, Proc. Euroconference on "The Impact of LargeSscale Near-Infrared Surveys", eds. F. Garzon, N. Epchtein, A. Omont, W.B. Burton, P. Persi, Kluwer Academic Publishers, Dordrecht, in press

Pérault M. et al., 1996, Astron.Astrophys. Lett., in press

Ruphy S., et al., 1996, Astron.Astrophys. Lett. 313, L21 